\title{
Determination of 3-Dimentional Interrogation Zone in Anti-collision RFID Systems with Inductive Coupling by Using Monte Carlo Method
}

\author{
P. JANKOWSKi-MihuŁOWICZ*, M. WęGLARSKI \\ Rzeszów University of Technology, Department of Electronic and Communications Systems \\ W. Pola 2, 35-959 Rzeszów, Poland
}

The operation of passive anti-collision RFID system is characterized by the interrogation zone which is estimated in any direction of 3D space for a group of electronic transponders. The elements of algorithm for determining the 3D interrogation zone in an inductively coupled anti-collision RFID system have been presented in this paper. The energy conditions have been analyzed in detail. The algorithm based on the Monte Carlo method and the computer program implemented in MathCAD engineering calculation software has been utilized in order to achieve the posed aims

PACS: $02.70 . \mathrm{Uu}, 41.20 . \mathrm{Gz}, 84.90 .+\mathrm{a}$

\section{Introduction}

The inductively coupled passive Radio Frequency Identification (RFID) system operates by direct coupling between the antenna units of the communication set consisting of the Read/Write Device (RWD) and electronic transponders [1]. Only one object identified by a single RFID transponder can be in the Interrogation Zone (IZ) according to the assumptions valid for basic solutions. Such an arrangement is called the single system and is most often used but also has limited possibilities in potential RFID applications. In the anti-collision system the communication process is carried out simultaneously with many RFID transponders (localized in the space $\left.\Omega_{I D}\right)$ by using algorithms of multiple access to the radio channel and communications protocols, just like in the standards operating at $13.56 \mathrm{MHz}$ : EPC (Electronic Product Code) Class-1 HF RFID Air Interface Protocol, ISO/IEC 15693, ISO/IEC 18000-3 normalizations (Fig. 1).

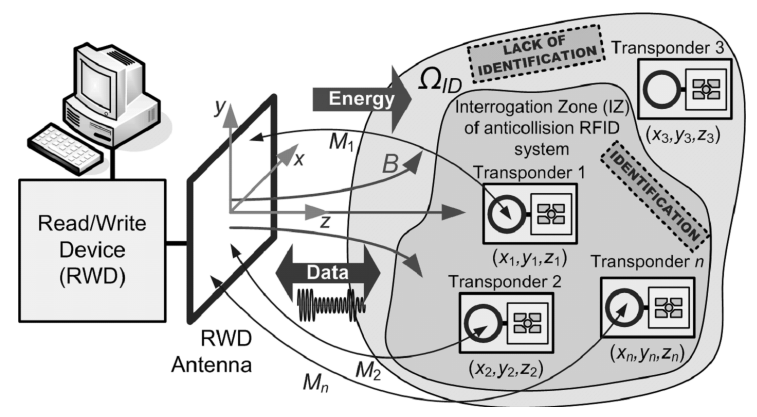

Fig. 1. Anti-collision RFID system with inductive coupling.

\footnotetext{
${ }^{*}$ corresponding author; e-mail: pjanko@prz.edu.pl
}

It is extremely difficult to simulate and design such anti-collision systems due to mutual interactions between antenna circuits of the RWD and transponders. Position changes and also problems with determination of variable communication and energy conditions cause these difficulties.

The inductively coupled RFID systems operate by using area in which an inhomogeneous magnetic field (characterized by the induction $\boldsymbol{B}$ or the magnetic field strength $\boldsymbol{H}$ ) and a strong coupling (characterized by the mutual inductance $M$ ) between antennas of the communication system built from the RWD and transponders occur. In these systems when determining the interrogation zone, the conditions for transferring energy to the passive transponders without internal power source are critical [2].

\section{Energy transfer in anti-collision RFID system}

A desired spatial distribution of many transponders in such a complicated system forces a synthesis of the three-dimensional (3D) interrogation zone in an unconventional way. This parameter is necessary not only in a simple stationary process of automatic identification but also for applications that act using dynamic changes of location and orientation of transponders on marked objects [3]. Therefore, a multi-aspect analysis of the inhomogeneous magnetic field generated by the antenna of the read/write device is required. The spatial position of each transponder antenna $\left(x_{1}, y_{1}, z_{1}\right) \div\left(x_{n}, y_{n}, z_{n}\right)$ should be taken into consideration independently due to the heterogeneity of magnetic field in the induction zone (the induction zone is considered as the near magnetic field according to the classical theory of antennas; it refers to all inductively coupled RFID systems). Furthermore, the passive transponder - all functional electronic blocks of its internal chip - is powered during a data transmission process according to a communication protocol. 
Minimum energy conditions must exist before beginning the communication. This energy is determined by the minimum value of magnetic field strength $H_{\min }$ (in the transponder location) at which the correct data transmission between the RWD and the transponder takes place. Their fulfilment is necessary for the proper operation of the whole anti-collision RFID system.

Investigations presented at the literature are concerned with increase in distance between centres of antennas but independently for each arrangement of RWD and single transponder. It leads to expanding the area (interrogation zone) in which transponders are able to operate according to requirements of an automatic identification process. In reality, only simple geometric configurations of antenna assemblies can be analysed in most cases [4, 5]. An influence of the transponder's antenna deflection is rarely analysed and additionally it is considered only in a one selected direction. Moreover, loops of the transponder antenna are located only in the symmetry axis of the RWD antenna in an analysis of this issue. It is not possible to take advantage of such geometrical arrangements in the vast majority of processes that require and anti-collision identification with variously placed and oriented transponders. Because of difficulties in identifying and describing (by analytical dependences) parameters affecting the operation of an passive transponder in the inhomogeneous magnetic field, there are only experimental methods of finding the proper location of transponders on marked objects in many cases of contemporary RFID system synthesises. These methods are very expensive and time consuming in practice.

\section{Determining the 3D interrogation zone using Monte Carlo method}

The presented premises cause the necessity of implementation of techniques which make use of random numbers. Their use is the basis for the Monte Carlo (MC) method [6]. A great interest in this method can be observed in recent years. It can be applied to solve some problems of RFID technology, for example in such areas as: location of objects [7-9], optimization of communication protocol parameters [10], data transmission [11, 12], elimination of collisions between the read/write devices [13], signal detection in RWD circuits [14] or determination of chip impedance in transponders [15].

The presented research problem concerns the development of $2 \mathrm{D}$ procedures (published in [16]) for testing the effectiveness of an antenna unit array in a system consisting of RWD and transponders, by using the MC method. This method has been applied to determine the 3D interrogation zone of an inductively coupled anti-collision RFID system. The effectiveness has been defined on the basis of the interrogation zone synthesis for a group of $n$-transponders which are located in the space $\Omega_{I D}$, and for a given automatic identification process (for example: Internet of Things [3]).

From a practical point of view of a planned implementation of anti-collision RFID systems, the most useful solution is to look for the interrogation zone with a given shape, position and orientation in space. The essence of its determination by the proposed method is presented in the Fig. 2.

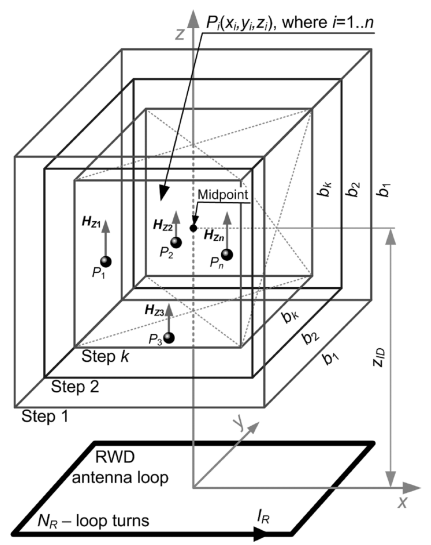

Fig. 2. Graphic representation of the process of determining the 3D interrogation zone in anti-collision RFID system by using MC method.

In the conducted research, it was assumed that the demanded volume should be cube shaped (of side $b$ ) and situated at the $z_{I D}$ height, whereas its location should be axially symmetrical and parallel to the RWD antenna loop. Such an assumption results from the orientation of transponders that are parallel to symmetrical RWD antennas which have, for example, a circular, square or different polygon shape in the inductive coupling RFID systems. The process of the interrogation zone determination is realized according to an algorithm presented in the Fig. 3.

A random deployment of $n$-transponders at $P_{i}$ points of Cartesian space at $\left(x_{i}, y_{i}, z_{i}\right)$ has been assumed in considered steps in a sequence - $k$ during the search of the RFID system interrogation zone. The random variables $x_{i}, y_{i}$ and $z_{i}$, for $i=1 \ldots n$ have a uniform distribution. It result from the fact, that electromagnetic field in any point of the communication space is heterogeneous. The random variables $x_{i}, y_{i}$ and $z_{i}$ are mutually independent.

The interrogation zone is determined for a given efficiency of identification $\eta_{I D}$ :

$$
\eta_{I D}=\frac{l_{\mathrm{IDOK}}}{n} \cdot 100
$$

where $l_{\text {IDOK }}$ is the number of transponders for which desired read/write operations have been properly done.

In order to determine that the RFID system is functioning correctly for given locations of transponders, it is not enough to achieve the $\eta_{I D}=100 \%$ for $n$ transponders and fulfill all conditions (absolute value of the $z$-component of magnetic field strength $H_{z} \geq H_{\min }$ ) for a correct operation of anti-collision RFID system. It cannot be predicted whether the sampling of coordinates of transponders placed in $k$ area in which all the conditions mentioned above are fulfilled meets the conditions necessary for correct operation of the whole RFID sys- 


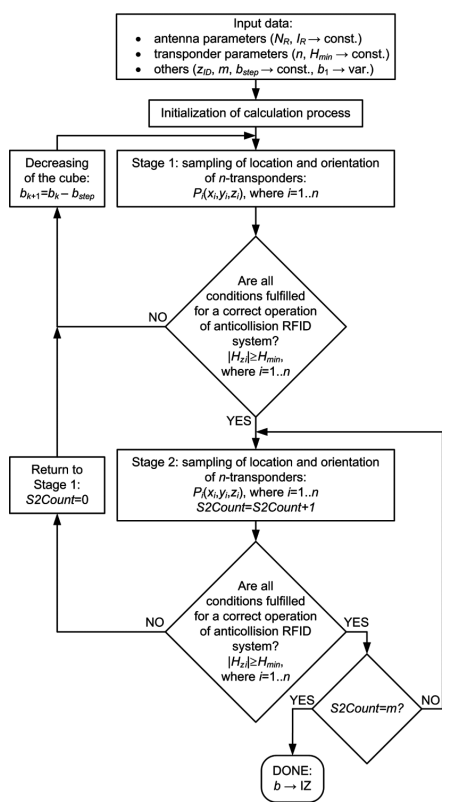

Fig. 3. Algorithm of the process of determining the 3D interrogation zone in anti-collision RFID system by using MC method.

tem with given efficiency. The practical use of the law of large numbers [6] for a $k$-step is a solution to this problem. It can be found that the $m$-tuple increase of the number of the random variables $x_{i}, y_{i}$ and $z_{i}$ sampling in $k$-step lengthens the calculation process during the simulation of an antenna unit arrangement. In accordance with the law of large numbers, the probability of a correct estimation of the interrogation zone increases. This is mainly connected with the examination of a larger number of deployed $n$-transponders cases. If the conditions of the correct operation of anti-collision RFID system are not fulfilled in any of $m$ multiple sampling of transponders location for $k$ analyzed area, then the next process of multiple sampling should be stopped, and it becomes necessary to examine the next $(k+1)$ - smaller cube $\left(b_{k}=b_{k-1}-b_{\text {step }}\right)$ of transponders location. The MC solution for the analyzed object completes a procedure which confirms the fulfillment of all conditions for the correct operation of anti-collision RFID system. The procedure is correct for a given efficiency of identification and for the area in which all the $m$ multiple sampling of transponders location lead to a positive calculation result of the antenna unit arrangement consisting of a $\mathrm{read} /$ write device and transponders.

\section{Results}

Elements of HF long range RFID systems with inductive coupling (working frequency: $f_{0}=13.56 \mathrm{MHz}$ ) have been used for testing the elaborated method of 3D interrogation zone determination. An analysis of the RWD antenna in HF RFID systems allows to make an assumption that the loop current $\left(I_{R}-\right.$ Fig. 2$)$ is constant along the whole flow path. In the case of RWD antenna loop constructed as polygon, Biot-Savart law with superposition theorem permits to add vectors at spatial location $P(x, y, z)$; the vectors descend from individual antenna parts [16]. The $z$-component of magnetic field strength in any point $P$ is given for the square loop shape by the following equation:

$$
\begin{aligned}
H_{z} & =\frac{I_{R} N_{R}}{4 \pi} \\
& \times\left(\int_{\frac{-a}{2}}^{\frac{a}{2}} \frac{y+\frac{a}{2}}{\left[\left(x-x^{\prime}\right)^{2}+\left(y+\frac{a}{2}\right)^{2}+z^{2}\right]^{3 / 2}} \mathrm{~d} x^{\prime}\right. \\
& +\int_{\frac{-a}{2}}^{\frac{a}{2}} \frac{-x+\frac{a}{2}}{\left[\left(x-\frac{a}{2}\right)^{2}+\left(y-y^{\prime}\right)^{2}+z^{2}\right]^{3 / 2}} \mathrm{~d} y^{\prime} \\
& +\int_{\frac{a}{2}}^{\frac{-a}{2}} \frac{y-\frac{a}{2}}{\left[\left(x-x^{\prime}\right)^{2}+\left(y-\frac{a}{2}\right)^{2}+z^{2}\right]^{3 / 2}} \mathrm{~d} x^{\prime} \\
& \left.+\int_{\frac{a}{2}}^{\frac{-a}{2}} \frac{-x-\frac{a}{2}}{\left[\left(x+\frac{a}{2}\right)^{2}+\left(y-y^{\prime}\right)^{2}+z^{2}\right]^{3 / 2}} \mathrm{~d} y^{\prime}\right)
\end{aligned}
$$

where $a$ is side length and $N_{R}$ - number of loop turns.

The experimental determination of the interrogation zone is divided into two parts: calculation and measurement. In both parts of the experiment, a laboratory process of anti-collision RFID identification has been subjected to verification. For the computational part a program called JankoRFIDmc'3D-IZ has been developed in MathCAD environment. This program enables a random deployment of $n$-transponders and to study the effectiveness of an antenna set. The elaborated algorithm presented in the Fig. 2 and Fig. 3 has been utilized in its source. The interrogation zone for a given efficiency of identification (defined by (1)) is the solution of this calculation. The result of numerical calculations by JankoRFIDmc'3D-IZ and the importance of its individual elements is presented in the Fig. 4 as an example.

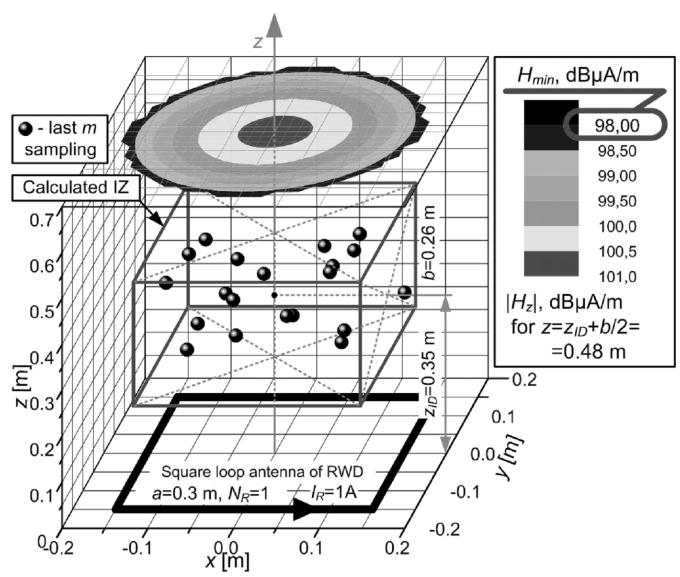

Fig. 4. Description of example elements of calculated 3D interrogation zone for TI RI-I11-112A-03 transponders $(n=20)$. 
The calculations have been performed for the following models: transponder RI-I11-112A-03 made by Texas Instruments $\left(H_{\min }=98 \mathrm{~dB} \mu \mathrm{A} / \mathrm{m}\right)$, square antenna $\left(a=0.3 \mathrm{~m}, I_{R}=1 \mathrm{~A}, N_{R}=1\right.$ coil [17]) supplied with terminals with $5 \mathrm{~W}$ from RWD ID ISC.LR200, by FEIG. The three-dimensional interrogation zone is characterized by the obtained cube with the edge length $b=0.26 \mathrm{~m}$ for $z_{I D}=0.35 \mathrm{~m}$. In Fig. 4 , the location of 20 transponders is marked for $m$-th sampling of $x_{i}, y_{i}$ and $z_{i}$, variables, for which all conditions of correct operation of RFID system have been fulfilled. In the upper part of the figure, a diagram of $\left|H_{z}\right|$ values is placed; it is determined for the most distant $x-y$ plane of the interrogation zone $\left(z=z_{I D}+b / 2\right)$. The obtained IZ is a result of positive identifications of all serial numbers of 20 transponders (read data for $\eta_{I D}=100 \%$ ) gathered during all 10000 sampling of their location. The calculations have been carried out with a step $b_{\text {step }}=0.01 \mathrm{~m}$. They have been started from the initial value $b_{1}=0.5 \mathrm{~m}$. Remaining results of the two stages of the experiment are summarized in the Table.

TABLE

Example of calculated and measured 3D IZ

\begin{tabular}{c|c|c}
\hline \hline$z_{I D}[\mathrm{~m}]$ & \multicolumn{2}{|c}{ Interrogation zone $(b)[\mathrm{m}]$} \\
\cline { 2 - 3 } & calculated & measured \\
\hline 0.15 & 0.30 & 0.28 \\
0.20 & 0.31 & 0.30 \\
0.25 & 0.33 & 0.32 \\
0.30 & 0.30 & 0.30 \\
0.35 & 0.26 & 0.25 \\
0.40 & 0.20 & 0.19 \\
0.45 & 0.14 & 0.14
\end{tabular}

The measured part of the experiment has been carried out in a specialized RFID laboratory of the Department of Electronic and Communications Systems of the Rzeszów University of Technology. Detail parameters of the models used in the calculations as an equivalent of RFID system components corresponded to the parameters of devices available in the laboratory. Measurements have been carried out in a special laboratory stand (Fig. 5). Experimental research in the operation of inductively coupled RFID system as well as in energy and communication conditions is possible to carry out in this stand.

The measuring chamber with moving platforms is the basic part of the laboratory stand. The passive RFID transponders were deployed in this chamber. The measured interrogation zone is the result of the positive identification of all serial numbers of transponders, during conducted experiment, for all 25 multiple sampling of their location. For control, for every multiple sampling of the location of transponders in chamber, spatial measurements of $z$-component of magnetic field strength $\mathbf{H}$ vector were made. A complete control of an automatic

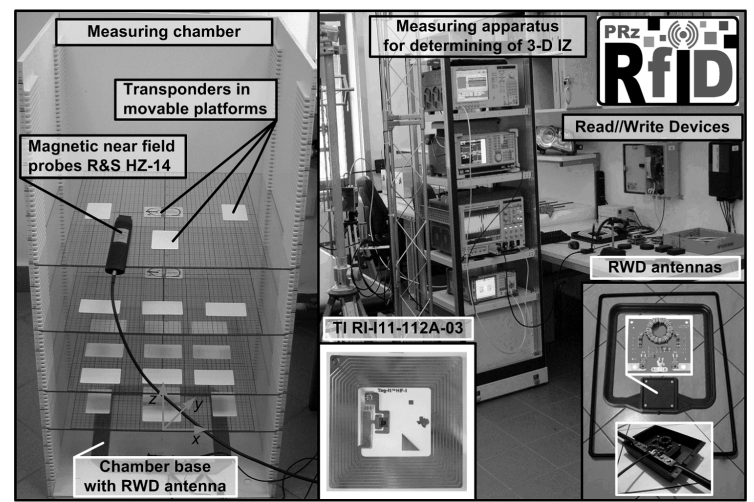

Fig. 5. Test stand in RFID Laboratory.

identification process in the field of energy and communication condition of operation of measured RFID system was possible thanks to the rich measuring equipment (oscilloscope Tektronix DPO71254B with probes P7504, CT1, TCP312+TCPA300, spectrum analyzer R\&S FSL18 with probes near-field HZ-14 and HZ-15, spectrum analyzer Tektronix RSA 3408B arbitrary waveform generator AWG5002B supplemented by vector generator R\&S SMBV100A).

\section{Conclusion}

The convergence of the measurements and calculations confirms a practical usefulness of the presented concept of determining the three-dimensional interrogation zone by using the Monte Carlo method in inductively coupled anti-collision RFID systems. The program JankoRFIDmc'3D-IZ developed on the basis of this method is practically used to solve many problems - reported by industry representatives - connected with implementation of RFID systems. It is possible to conclude on the basis of realised work that the parameters of antenna set in arrangement of RWD and transponders are the most important factor determining the effectiveness of radio identification process for multiple objects simultaneously. Taking into consideration the usefulness of the interrogation zone in a given process, it is necessary to permanently posses a knowledge base about the parameters of RFID devices introduced into the market.

\section{Acknowledgments}

This work was partly supported by the Projects: "Developing research infrastructure of Rzeszów University of Technology" within the Operational Program Development of Eastern Poland 2007-2013 of the Priority Axis I Modern Economics of Activity I.3 Supporting Innovation, Contract No. POPW.01.03.00-18-012/09-00; "Developing and modernization research base of Rzeszów University of Technology", No UDA-RPPK.01.03.00-18003/10-00 from The Structural Funds, The Development 
of Podkarpacie Province, The European Regional Development Fund.

\section{References}

[1] K. Finkenzeller, RFID Handbook, 3-rd Ed., Wiley, 2010.

[2] P. Jankowski-Mihułowicz, Elektronika, 8, 63 (2010).

[3] E. Jones, C. Chung, RFID in Logistics - A Practical Introduction, CRC, 2008.

[4] B. Jiang , J.R. Smith, M. Philipose, S. Roy, K. Sundara-Rajan, A.V. Mamishev, IEEE Trans. Instrum. Meas., 56, 118 (2007).

[5] Se-gon Roh, Hyouk Ryeol Choi, IEEE Trans. Autom. Sci. Eng., 6, 55 (2009).

[6] D.P. Kroese, T. Taimre, Z.I. Botev, Handbook of Monte Carlo Methods, Wiley, 2011.

[7] T. Lindgren, B. Kvarnstroöm, J. Ekman, IET Microwaves, Antennas \& Propagation, 4, 2069 (2010).

[8] Yun Bae Kim, Jinsoo Park, Seho Kee, Kiburm Aong, Mi Ae Han, Proc. of Winter Simulation Conf, 1957 (2010).

[9] Junyi Zhou, Jing Shi, Xiuli Qu, Int. J. Adv. Manuf. Technol., 46, 1215 (2010).
[10] D.K. Klair, Kwan-Wu Chin, R. Raad, Proc. of IEEE Int. Symp. of Communications and Information Technologies, 1401 (2007).

[11] Thanh T. L. Tran , A. Mcgregor, Yanlei Diao, Liping Peng , Anna Liu, Proc. of the VLDB Endowment, 3, 1302 (2010).

[12] Junyi Xie, Jun Yang, Yuguo Chen, Haixun Wang, P.S Yu., Proc. of 24th Int. IEEE Conf. on Data Engineering, 476 (2008).

[13] Haiquan Chen, Wei-Shinn Ku, Haixun Wang, MinTe Sun, Proc. of the Int. Conf. on Management of Data, 51 (2010).

[14] J.-H. Bae, W.-K. Choi, J.-S. Kim, G.-Y. Choi, J.S. Chae, Progress In Electromagnetics Research, 91, 243 (2009).

[15] T. Bjorninen, M. Lauri, L. Ukkonen, R. Ritala, A.Z. Elsherbeni, L. Sydanheimo, IEEE Trans. Instrum. Meas., 60, 3194 (2011).

[16] P. Jankowski-Mihułowicz, W. Kalita, Current Trends and Challenges in RFID, Ed. C. Turcu, Intech, 335 (2011).

[17] P. Jankowski-Mihułowicz, Elektronika, 8, 73 (2010). 